\title{
Software of passenger vehicle optimal work and energy recovery (POWER)
}

\author{
H. Farzaneh ${ }^{1} \&$ Y. Saboohi ${ }^{2}$ \\ ${ }^{1}$ Department of Environment and Energy, Science and Research Campus, \\ Azad University, Tehran, Iran \\ ${ }^{2}$ Sharif Energy Research Institute, Sharif University of Technology, \\ Tehran, Iran
}

\begin{abstract}
In our investigation, the model of optimal energy flow in a passenger vehicle has been founded on the theory of firm microeconomics. Based on this theory, the car owner tries to minimize the total cost of the system (including the cost of time of the traveller) subject to the satisfaction of the required transport services and technological, economical, environmental and institutional constraints. The aforementioned model has been developed using a technique of mathematical programming. The model depicts the behaviour of a nonlinear system and it includes many nonlinear functions in the objective function and in the constraints. Solving the large nonlinear set of constraints and identifying the global optimal energy flow was a major issue in the process of developing the model. Therefore, an integrated approach based on numerical analysis, linear programming and the concept of control volume, as a means of defining open and interrelated systems, has been developed and applied in solving the model. Solution of the model has been based on the boundary conditions that define the surroundings of the vehicle. The output data resulting from solution of the model are: material elements and optimal energy balances in different parts of the vehicle, transient behaviour fuel consumption and emission of pollutants in the course of operation of the vehicle.
\end{abstract}

Keywords: passenger vehicle, energy flow optimization, mathematical programming. 


\section{Introduction}

Urban passenger transport is a major energy consuming sector and it has tremendous impact on environmental pollution. The effect of urban passenger transport on the energy consumption and environmental pollution is more acute in developing countries than in industrialized countries, which may be due to the lack of reliable and effective mass transport infrastructure in these countries. Contribution of private passenger vehicles to the transport services is, therefore, considerable. Improvement of energy efficiency and regulation of emission of pollutants from passenger vehicles could provide an effective means of easing the negative economical and environmental impact of urban transport systems. Technology assessment and efficiency evaluation of passenger vehicles have been pursued with the help of analytical tools that have been developed and implemented in the last two decades. Many of the available analytical tools are based on the heuristics and experimental data. But there has been little effort on concluding the theoretical background of analysis and developing respective energy models according to the optimal behaviour of the system. Based on this conclusion, software of passenger vehicle optimal work and energy recovery (POWER) has been developed.

In the POWER software, a passenger vehicle is supposed to be organised in the form of a firm and appears in the market as a supplier of goods and commodities. The activity of a producer is usually oriented towards establishing an effective supply system which may be identified as delivering the product with minimum costs. The economic rationality of a producer and activity of a firm has extensively been developed as a branch of micro economics and it has been utilised for explaining the development of production technology. It is clearly stated that a firm strives towards establishing a system of production and delivery with minimum costs subject to satisfying the demand and other technical, economical and institutional constraints.

Application of the POWER is subject to the identification and estimation of the person per mileage removable as a production function. Difficulty in obtaining an explicit form of the production function has in many cases led to an indirect solution of the model. This is usually achieved by defining person per mileage as a function of parameters of the model (i.e. prices of production factors, demand and bounds on the production).

Production function represents a nonlinear system and it is a complex function. Empirical estimation of the complex function would be associated with considerable over or under estimations. It is, therefore, possible to segregate the passenger vehicle system into incremental elements and substitute the nonlinear production function with a set of interrelated equations. Simultaneous solution of the set of equations would help identifying the function of the whole system and state of variables at different points of the system and time can be estimated. Evaluation of the function of the system at a segregated level and at different time points provides appropriate means of studying the impact of various factors on the development of the vehicle system. 


\section{Software structure}

POWER software is an analysis tool for energy flow optimization in a passenger vehicle that was created in Fortran/Visual Basic programming. Fortran codes provide a matrix-based programming environment for performing calculations based on the concept of a model while Visual Basic code is used to represent a user friendly interface for a usual user. POWER uses graphical user interface screens to guide the users through the modeling process. With the interface section, the user can iteratively evaluate the impacts of vehicle parameters and drive cycle requirements on the vehicle performance, economy, environment, traffic and Iinstitutional conditions. The interface facilitates interaction with the raw input and output data that is present in the Fortran codes and the vehicle energy model is depicted graphically to define the connections between components. The model reads the input data from the prepared powerful database during the modeling and optimization and outputs the results to the workspace to be viewed in the output reports window. In the POWER input data windows shown in fig. 1, the user builds the vehicle boundary conditions. The vehicle boundary is divided into 5 sections as follows:

- Time Horizon: is a dimension of the system that depicts dynamics and the path of operation of the vehicle that is represented along the time dimension.

- Vehicle Levels: are an integration of sub-systems and their interactions representing the structure of the vehicle. The whole system of the vehicle is segregated into sub levels and the flow of material and energy encompasses the interrelationship between control volumes. Vehicle levels and flow of energy and materials indicate structural dimensions of the vehicle.

- Load Curve: in addition to the time dimension, it is also observed that the flow of energy and materials varies within the defined time points. As a result, flow rate variations are characteristic of the chosen drive cycle. The load curve, therefore, represents the variation of energy flow rate within a time point such as 4 stroke engine processing in the vehicle.

- Vehicle Structure design: is a graphical possibility for structuralization the total vehicle system and definition of the control volume as the vehicle components.

Demand and output request of the model is defined in the system environment section. As described previously, person per mileage defines the expected output request of the model based on the optimization procedure in the software. Specific technical constraints such as pollutions and traffic limitation are an indispensable part of the vehicle system. The limit on the quality of material at each level and combination of the elements of the flow and the costs associated with the limits are the main data required for detailed and comprehensive analysis of development of energy flow in the vehicle.

The vehicle is composed of various sub-systems and control volumes provide the basis of the structural development of the system of the vehicle. 
Development of the vehicle system may be studied through analysis of the functions of control volumes and the interactions between them. It is therefore necessary to define the dimensions and functions of the control volumes and relationships between them. The major categories of data representing the specifications of control volumes and the structure of the whole vehicle system are depicted in fig. 1.

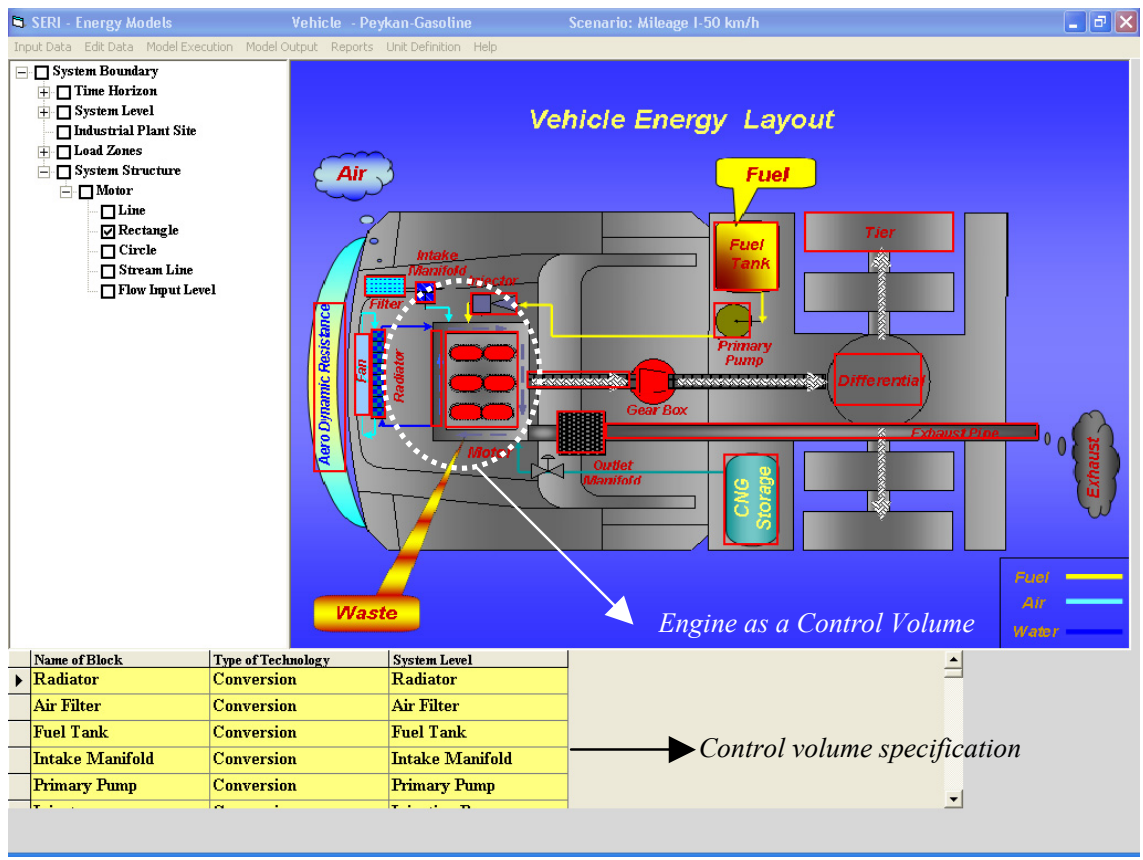

Figure 1: Input data window, vehicle structure and control volumes definition.

Technical aspects and system function are defined in the vehicle system technologies sub-window of input data window. Conversion processing depicts the flow of material/energy through an open system and its behavior is conditioned by the basic laws of physics and thermodynamics. In addition to that, development and operation of the vehicle undergo various technical, economical and institutional constraints. The control volumes are an open system and energy and material flows through them. Three main categories of open control volumes are defined in the software structure. They represent processing/conversion components (Engine, Gearbox, Differential, Radiator, Air filter, Intake manifold, injector or carburetor, outlet manifold and wheels), header (fuel linkage, electric network and lubrication system) and storage (fuel storage and battery) as depicted in fig. 2 .

The list of inputs is defined as the energy/material carriers in the last system level in the section of the system boundary. Type of fuel and its price are defined 
in this section. In addition, a special limitation on the fuel supply such as Iinstitutional conditions can be considered as input to the plant sub-window.

When the actual vehicle is composed of a collection of boundary, environment, technologies and input to the plant data, it can be stored in the database of the model. The collection data can be inserted into the model to connect the defined control volumes to establish energy flow from one component to the next.

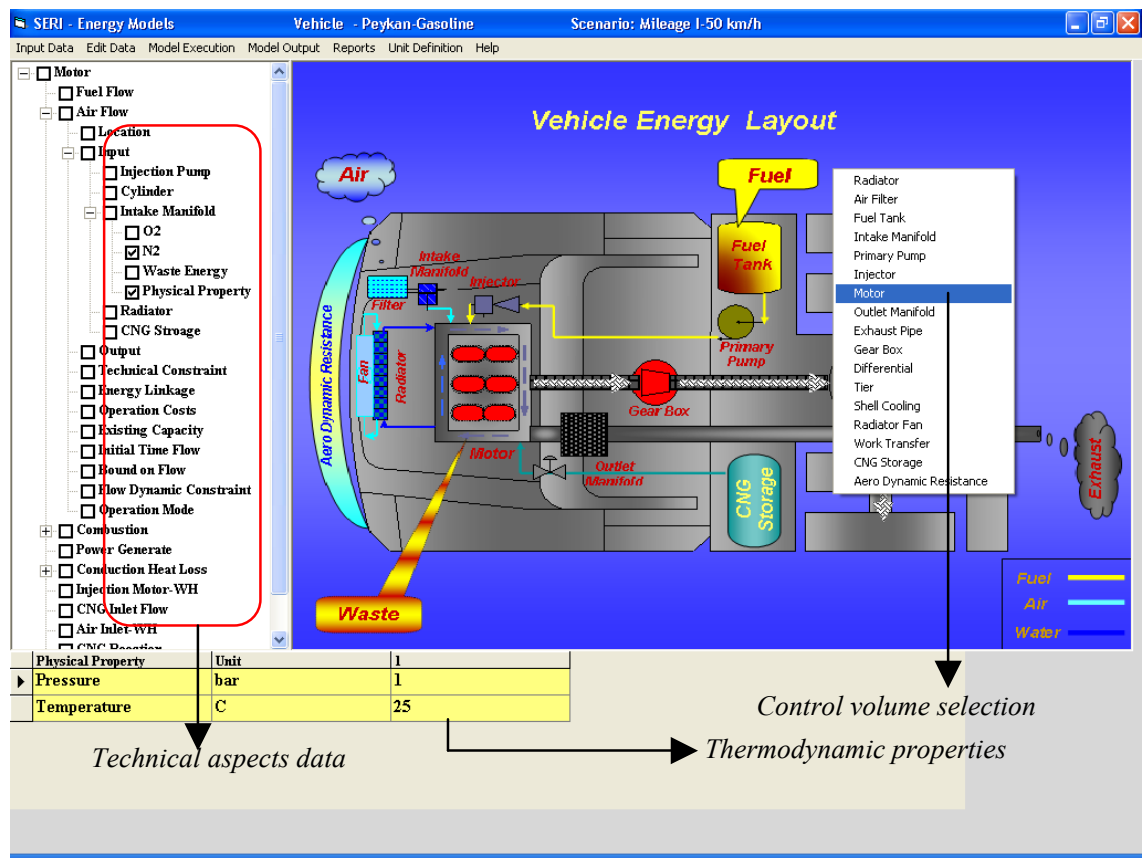

Figure 2: Input data window, vehicle technical aspects definition.

The model library approach allows the same component model to be reused in multiple vehicle configurations. It also allows the impacts of different models to be evaluated within a single vehicle architecture. After processing of data, the library is linked to Fortran codes (Matrix generator and optimizer) and then optimal results will be observed through the output report window depicted in fig. 3.

Output results of the model are categorized in 8 parts:

- Mass and energy balances in different control volumes.

- Supply and demand match.

- Optimal energy flow and capacity profile of each control volume.

- $\quad$ Storage profile in each load zone.

- Energy flow networking and distribution in total site of vehicle

- Total system and marginal cost of vehicle movement.

- Resources intensities and effectivities.

- Scenario comparison. 


\section{Software approach}

POWER software using a backward facing approach answers the question "assuming the vehicle met the required person per mileage mobility, how must each component conform and how mush energy will be required".

The force required to accelerate the vehicle through the time step is calculated directly from the required speed trace.

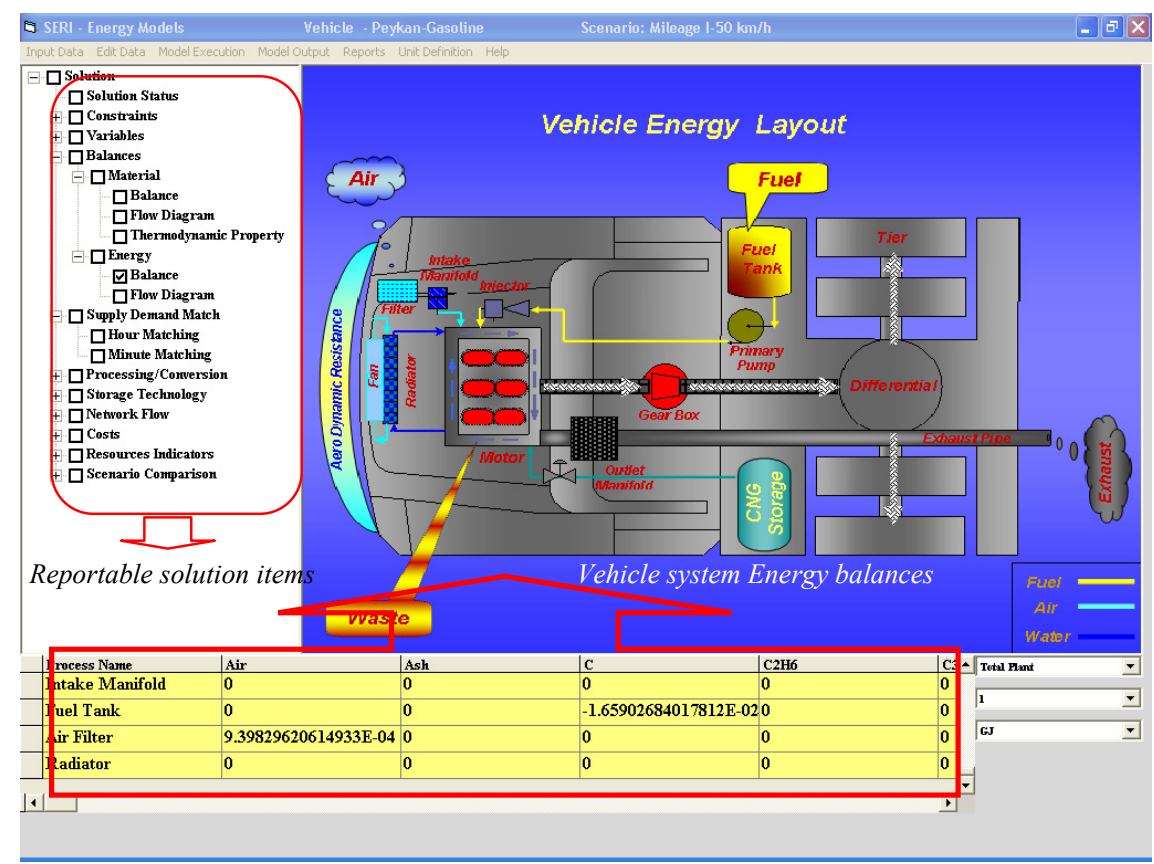

Figure 3: Output report window, vehicle energy balances.

The required force is then translated into a torque that must be provided by the component upstream, and the vehicle's linear speed is likewise translated into the required rotational speed and the generated engine power. Component by component, this calculation approach carries the energy backwards through the drive train, against the traction power flow direction, until the optimal fuel and electrical energy use that would be necessary to meet the demand through the optimization algorithm is computed.

This approach is convenient because automotive driveline components tend to be tested with a standard design specification of each one and allows very simple integration routines between each component to the next. 


\section{Results and discussion}

In this section the optimal results of the model of passenger vehicle optimal work and energy recovery (POWER), as described in the previous section, are presented.

The initial data required to construct the boundary conditions of the model for a case study contains the following groups that are depicted in table 1:

- Estimated mileage and person per mileage of chosen cycle (Urban and Highway)

- Weather condition (temperature, pressure and wind speed).

- Design specifications of vehicle (weight, dimension, technical components)

- Fuel price and environmental taxes.

- Iinstitutional conditions (permitted velocity and prohibited passage).

The required power for vehicle movement is a function of the traction force at the various ranges of the speed trace. As we know, the traction force is a function of the physical properties of the road and weather and also the dimensions of the vehicle. The chosen cycle is divided into urban and highway sections. It is viewed in fig.3; because of traffic and institutional limitations, the vehicle moves through an urban section with $1^{\text {st }}$ to $2^{\text {nd }}$ gear and through the highway with $3^{\text {rd }}$ to $4^{\text {th }}$ gear.

Table 1: Input data for execution of the model (case study).

\begin{tabular}{|l|l|}
\hline Cycle Condition : & Vehicle design specifications : \\
Duration $: 7813.7 \mathrm{~s}$ & Four stroke engine $1600 \mathrm{cc}$ \\
Mileage: $308.2 \mathrm{~km}$ & Net weight $: 1 \mathrm{kN}$ \\
Minimum velocity $: 10 \mathrm{~km} / \mathrm{h}$ & Fuel storage capacity : 45 liter \\
Maximum velocity: $142 \mathrm{~km} / \mathrm{h}$ & Fuel type : Petrol \\
& Gear Ratio: $3.35,2.14,1.39,1$ \\
& Axel Ratio: 2.81 \\
\hline Ambient condition: & Vehicle weight distribution : \\
Temperature $: 250 \mathrm{C}$ & Travelers $: 4$ persons \\
Pressure $: 1 \mathrm{~atm}$ & Travelers average weight $: 0.05 \mathrm{kN}$ \\
Wind velocity: $3 \mathrm{~m} / \mathrm{s}$ & Front weight $: 0.252 \mathrm{kN}$ \\
& Rear weight $: 0.948 \mathrm{kN}$ \\
\hline
\end{tabular}

The engine power is generated according to the transmission of the traction force through the differential and gearbox with a backward-facing approach. As fig. 3 shows, variance of the traction and engine power in the highway is more than the urban cycle and it can justify augmentation of resistance forces.

Optimal fuel consumption is calculated by the model in different speed durations along the mileage of the chosen cycle. It is viewed in fig. 4; the fuel consumption decreased from the urban condition to the highway because of the change of gear ratio. This can justify that the effect of gear ratio is more than the 
increased resistance force at high speed. The trend of fuel consumption has decreased until the observed circle mark that will be the start point of movement with $4^{\text {th }}$ gear at $85 \mathrm{~km} / \mathrm{h}$. This finding is consistence with the results obtained in Ref. 3.

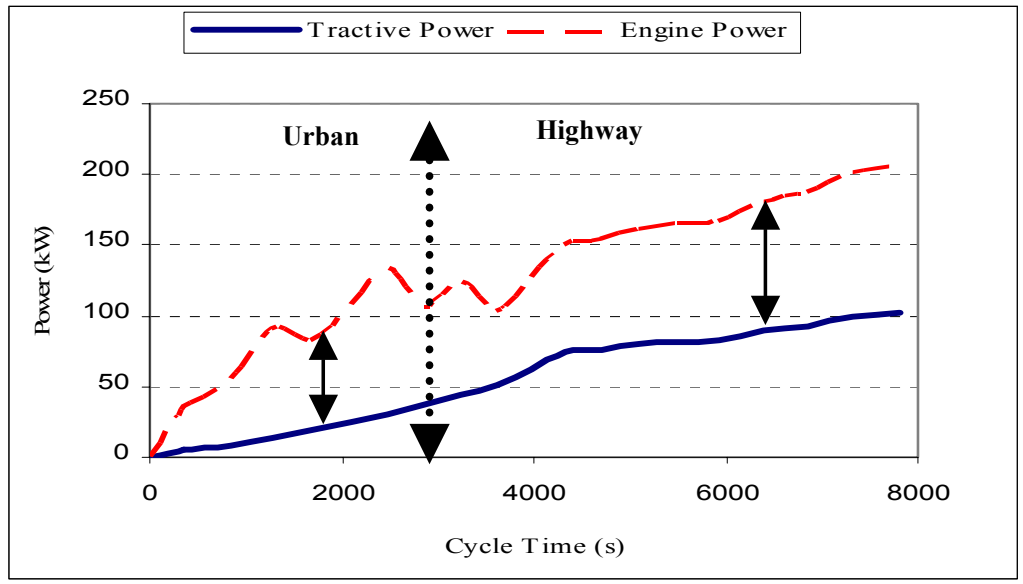

Figure 4: Variation of the traction and engine power during the time horizon of the chosen cycle.

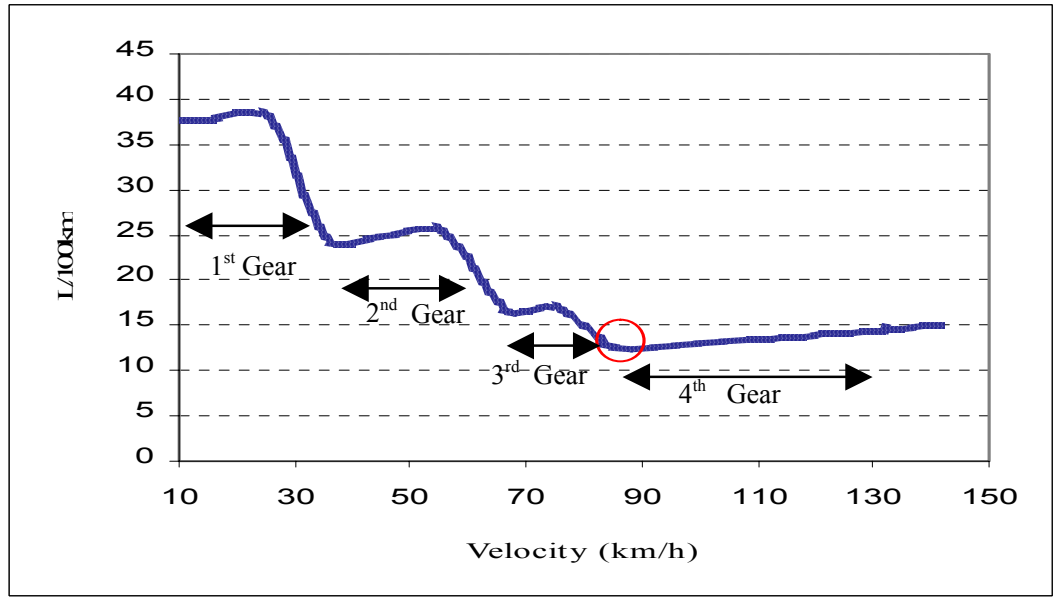

Figure 5: Comparison of optimal fuel consumption.

Fig. 6 depicts a comparison of the Traverse mileage per gallon (MPG) with total cost. The maximum point of the MPG coincides with the minimum point of the total cost that depicted excellent allegiance of the cost function and the fuel consumption. It is thought that the optimal point of the vehicle operation is obtained when the total cost of operation is minimized. 


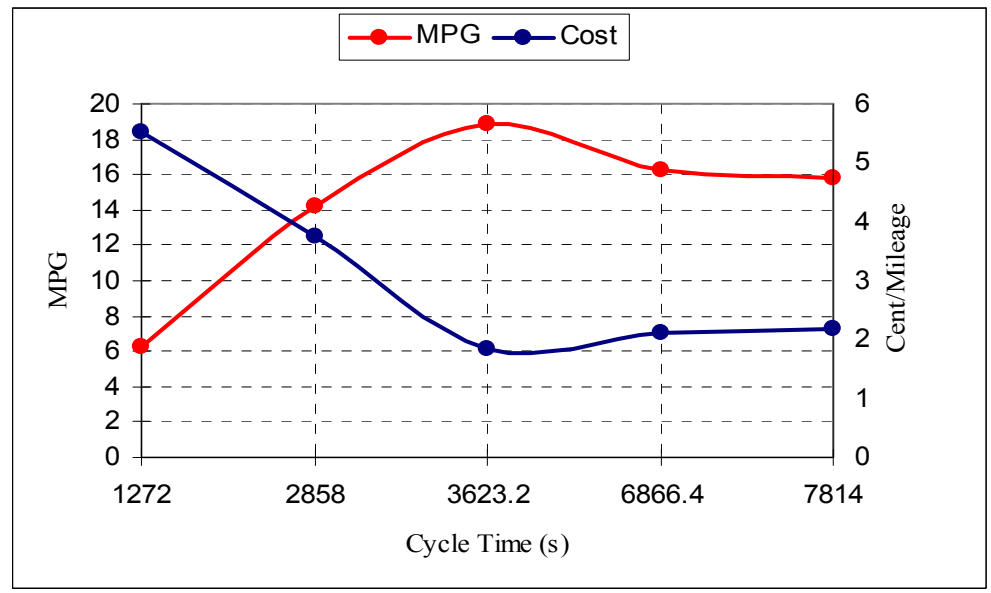

Figure 6: Comparison of optimal MPG and total cost during the time horizon of the chosen cycle.

\section{References}

[1] Saboohi, Y. Energy flow optimization model in industries, Sharif Energy Research Institute, 2000

[2] Olson, B.J., Nonlinear dynamics of longitudinal ground vehicle traction, Michigan state university, 2001

[3] Migaard, P., Mauss , F., Homogeneous charge compression engine : A simulation study on the effects of INHOMOGENEITIES, ICE-Vol. 34-2, 2000 Spring Technical Conference, pp 275, 283, ASME 2000

[4] Ogink, R., Gasoline HCCI modeling, Ph.D Thesis, Chalmers University of Technology, 2002

[5] SAYPA, Laboratory of dynamometric and road test of passenger vehicle, Result NO: L-S-10654-7, R\&D center

[6] Guezennec, Y.,G., Fundamentals of internal combustion engine, Center for Automotive Research and Intelligent Transportation, Ohio State University, 2003

[7] Nam, E., Advanced technology vehicle modeling in PERE, EPI United Stat, Environmental Protection Agency, 2004

[8] Cox, M., Bertness, K., Vehicle-Integrated Battery and Power System Management based on Conductance Technology to Enable Intelligent Generating Systems,pp1-6, Society of Automotive Engineering Inst., 2002

[9] Mrkel, T., Brooker, A., Hendricks,T., Wipke,K., ADVISOR: a systems analysis tool for advanced vehicle modeling, Journal of Power Sources, pp 255-266, 2002 